\title{
Plataforma Eco+: Habilitando o Comportamento Ecossustentável das Pessoas
}

\author{
Eco+ Platform: Enabling People's Eco-sustainable Behavior \\ Katyeudo Karlos Sousa Oliveira ${ }^{* 1}$, Ariany Silva Ferreira ${ }^{1}$, José Rafael Santana ${ }^{1}$, Vanessa Bandeira Teixeira ${ }^{1}$, \\ Ricardo André Cavalcante Souza ${ }^{1}$ \\ ${ }^{1}$ Universidade Federal Rural de Pernambuco, Pernambuco, Brasil.
}

\begin{tabular}{|c|c|}
\hline INFO ARTIGO & RESU MO \\
\hline $\begin{array}{l}\text { Palavras-chave: } \\
\text { Desenvolvimento } \\
\text { Sustentável, } \\
\text { Design Centrado no } \\
\text { Humano, } \\
\text { Mudança Climática. }\end{array}$ & $\begin{array}{l}\text { O desenvolvimento sustentável só é possível pela interconexão harmônica } \\
\text { entre crescimento econômico, inclusão social e proteção ambiental. A } \\
\text { Organização das Nações Unidas (ONU) definiu 17 Objetivos de } \\
\text { Desenvolvimento Sustentável (ODS) que devem guiar iniciativas de países e } \\
\text { sociedade civil até } 2030 \text {. Entre as metas do ODS } 13 \text {, está "aumentar a } \\
\text { conscientização e a capacidade humana sobre mitigação e redução do } \\
\text { impacto da mudança do clima". Assim sendo, o objetivo desse trabalho é } \\
\text { relatar a aplicação do Design Centrado no Humano para o desenvolvimento } \\
\text { de uma solução de inovação social motivada pelo seguinte desafio: "Como } \\
\text { incentivar as pessoas a adotar um comportamento ecossustentável?". O } \\
\text { resultado foi o design da plataforma Eco+, que fornece suporte tecnológico } \\
\text { aos seguintes processos: o usuário poderá realizar a coleta seletiva de resíduos } \\
\text { e então levar até estações responsáveis por destiná-los à compostagem e/ou } \\
\text { reciclagem; e o usuário poderá usar bicicletas adaptadas para transformação } \\
\text { da energia cinética e armazenagem da energia elétrica resultante. }\end{array}$ \\
\hline
\end{tabular}

ARTICLE INFO

\section{Keywords:}

Sustainable Development, Human-Centered Design, Climate Change.

\begin{abstract}
A B S T R A C T
Sustainable development is only possible through the harmonious interconnection between economic growth, social inclusion and environmental protection. The United Nations (UN) has defined 17 Sustainable Development Goals (SDGs) that should guide initiatives by countries and civil society until 2030. Among the goals of SDG 13 is "awareness-raising and human capacity on climate change mitigation and impact reduction". Therefore, the objective of this work is to report on the application of Human Centered Design for the development of a social innovation solution motivated by the following challenge: "How to encourage people to adopt eco-sustainable behavior?". The result was the design of the Eco + platform that provides technological support to the following processes: the user will be able to carry out the selective collection of waste and then take it to stations responsible for destining it for composting and / or recycling; and the user will be able to use bicycles adapted for the transformation of kinetic energy and storage of the resulting electric energy.
\end{abstract}

\footnotetext{
* Correspondência para autor:

karlos.1914.so@gmail.com (Oliveira, K.K.S.), ariany.ferreira@ufrpe.br (Ferreira, A.S.), joserafael.santana@ufrpe.br (Santana, J.R.), vanessa.bandeira@ufrpe.br (Teixeira, V.B.), ricardo.cavalcante@gmail.com (Souza, R.A.C.).
} 


\section{Introdução}

De acordo com o Painel Intergovernamental sobre Mudanças Climáticas (IPCC, 2007), mudança climática é uma alteração significativa dos parâmetros climáticos (como temperaturas ou precipitações) médios ou na sua variação em um extenso intervalo de tempo. A mudança climática pode ter suas causas justificadas por ações naturais da própria Terra ou por ações externas, incluindo variações na intensidade da luz solar, ou por ações antrópicas (provocada pelo homem). Entre as implicações mais relevantes das mudanças climáticas, pode-se citar o aquecimento global, a desertificação, e o aumento da temperatura das águas marinhas.

A mudança climática é um objeto de estudo complexo, com relações sociais e ecológicas substanciais. Para tratar sobre o tema, é indispensável adquirir habilidades para solucionar problemas e tomar decisões importantes em diversos níveis, desde a comunidade científica até o cidadão. Para decidir sobre as questões que evolvem as mudanças climáticas, é necessário conhecer as causas que as provocam e a contribuição das ações cotidianas que afetam o problema (OLIVEIRA; SOUZA, 2020).

As mudanças climáticas são temas centrais dos principais debates globais. Os formuladores de politicas e cientistas ainda têm uma visão limitada do que deve ser debatido sobre o tema (MOCHIZUKI; BRYAN, 2015). Tomar medidas urgentes para combater a mudança climática e seus impactos é um dos Objetivos de Desenvolvimento Sustentável (ODS 13) contido na Agenda 2030 da Organização das Nações Unidas (ONU, 2015). Entre as metas do ODS 13, está "aumentar a conscientização e a capacidade humana sobre mitigação e redução do impacto da mudança do clima". De acordo com Oliveira e Souza (2020), as mudanças climáticas podem resultar em complicações desde o bem-estar pessoal até a sustentabilidade dos negócios.

Segundo Visintainer e Linn (2015), são necessárias abordagens que possuam inovação e sejam sociais, para que assim, sejam obtidas soluções com capacidade de mitigação dos impactos das mudanças do clima. De acordo com Baregheh, Rowley e Sambrook (2009), inovação é o processo de multiestágio em que as organizações transformam ideias em produtos, serviços ou processos novos ou melhorados, assim, sendo possível avançar, competir e diferenciar-se com sucesso no seu mercado de atuação. Conforme Mulgan (2012), uma inovação social é uma inovação que é social tanto nos seus fins como nos seus meios, ou seja, cobre novas ideias que simultaneamente atendem necessidades sociais reconhecidas e criam novas relações ou colaborações sociais, sendo boas para a sociedade e aumentando a capacidade das pessoas agirem.

As abordagens inovadoras têm como características comuns serem centradas nas pessoas e baseadas na criatividade. Entre as abordagens inovadoras de referência, está o Design Centrado no Humano (HCD do inglês Human-Centered Design) (IDEO, 2015). O HCD pode ser considerado uma sistematização do design thinking (BROWN, 2009) e é composto pelas seguintes etapas: Inspiração, para entendimento aprofundado de um problema e elaboração de um desafio para orientar o projeto; Ideação, para geração, refinamento e combinação de ideias de solução e seleção daquela mais promissora para responder ao desafio proposto; e Implementação, para materialização da ideia de solução por meio de protótipo, visando avaliação desta junto aos usuários. No contexto deste trabalho, o objetivo não é a implementação de um produto final, apenas de um protótipo para obtenção de feedback sobre a solução proposta.

Tendo em vista que a ação antrópica é uma das causas das mudanças climáticas, o objetivo desse trabalho é relatar a aplicação do HCD para o desenvolvimento de uma solução de inovação social motivada pelo seguinte desafio: "como incentivar as pessoas a adotar um comportamento ecossustentável?". O resultado consistiu na prototipação e avaliação de um aplicativo de software denominado Plataforma Eco+, cuja finalidade é prover meios para a população utilizar cotidianamente alternativas sustentáveis visando o benefício coletivo, incentivada por um sistema de recompensas.

Além desta introdução, o trabalho está estruturado em mais três seções. A Seção 2 descreve a fundamentação teórica do trabalho. A Seção 3 descreve as etapas de desenvolvimento da solução proposta. A Seção 4 apresenta as considerações finais.

\section{Fundamentação Teórica}

De acordo com Phills, Deiglmeier e Miller (2008), uma inovação social deve ser justa e sustentável, tanto ambientalmente quanto para se manter e ser utilizada a longo prazo. Uma inovação social não deve focalizar somente nas ambições privadas (valores econômicos ou financeiros), mas produzir melhoria pública (valor social ou mitigar prejuízos sociais). Os valores sociais estão ligados, por exemplo, ao aprimoramento da educação, cultura, saúde, justiça e meio-ambiente. 
Uma inovação social é motivada por uma necessidade social, assim, a Agenda 2030 da ONU enumera diversas necessidades sociais, entre as quais aquelas relacionadas às mudanças climáticas (ONU, 2015). A evolução tecnológica possibilitou à humanidade avanços em todas as áreas, porém existe a indispensável necessidade de encontrar soluções capazes de auxiliar a redução de desigualdades sociais e a destruição ambiental, possibilitando a conservação da vida no planeta (ANASTACIO; CRUZ FILHO; MARINS, 2018).

As tecnologias digitais têm o potencial de aumentar a escalabilidade e a extensão dos beneficiados pelas inovações sociais. Deste modo, emergiu o conceito de Inovação Social Digital (DSI - Digital Social Innovation), que pode ser definido como um tipo de inovação social na qual inovadores, usuários e comunidades colaboram por meio de tecnologias digitais para cocriar conhecimento e soluções para uma grande faixa de necessidades sociais em uma escala e velocidade que eram inimagináveis antes do advento da Internet (BRIA et al., 2015).

Para o desenvolvimento de uma solução DSI, faz-se necessária a utilização de uma abordagem de inovação tal como o HCD. O HCD é uma das abordagens que atualmente estão sendo mais aplicadas na produção de inovações no campo social (ANASTACIO; CRUZ FILHO; MARINS, 2018; LIEDTKA; SALZMAN; AZER, 2017).

O HCD propõe um método enxuto para usar a criatividade em prol do desenvolvimento de soluções centradas nas reais necessidades das pessoas, a partir da execução de três etapas:

(1) inspiração, para imersão no contexto do problema visando o aprendizado, entendimento aprofundado das necessidades das pessoas e identificação de um desafio relevante;

(2) ideação, para dar sentido naquilo que foi aprendido anteriormente, identificar insights e gerar ideais para atender ou explorar tais oportunidades;

(3) implementação, para materializar e avaliar a ideia de solução que se mostrou mais promissora para resolver o desafio.

A partir de buscas na literatura especializada, foi possível encontrar estudos correlatos ao trabalho desenvolvido. Em Ribeiro et al. (2018), é relatado o uso das ferramentas do Toolkit do HCD no desenvolvimento de um aplicativo de alerta de alagamentos para atender necessidades sociais causadas pela ocorrência de chuvas, como mobilidade urbana, riscos de danos materiais e perdas de vida humana.

No estudo de Silva et al. (2019), os autores descrevem a aplicação do HCD para desenvolvimento de uma solução baseada em tecnologia digital para atender à necessidade social por uma comunicação tempestiva de informações do tempo para cidadãos e entre organizações públicas responsáveis pelo controle dos dados e por atuar em caso de eventos naturais.

Moulaert et al. (2007) descrevem um conjunto de soluções de inovação social que visam melhorar a quantidade e qualidade de vida de cidadãos europeus. Os autores comparam os discursos de inovação neoliberal e social sobre mudança socioeconômica urbana, incluindo políticas associadas e agências-chave. Após, procuram aprimorar o quadro analítico do desenvolvimento urbano combinando a Teoria da Regulação com elementos da Economia Política Cultural e da Teoria do Regime Urbano. Por fim, com o auxílio de estudos de caso, ilustram como a inovação social é um conceito com expressiva capacidade de apoiar os movimentos de mudança urbana.

Esse trabalho se diferencia dos demais analisados, pelo fato de ser motivado pelas consequências das mudanças climáticas causadas pela atividade humana. Ainda, possui a contribuição técnica de implementar o design de um aplicativo de software para habilitar a inovação social, através de estímulos de recompensas ao comportamento mais ambientalmente sustentável das pessoas.

\section{Metodologia de execução do processo HCD}

No contexto deste trabalho, as etapas do HCD foram executadas por meio de iterações denominadas sprint. Cada sprint possuía um objetivo específico e um prazo (time box) não prorrogável de dez dias corridos para conclusão. Para alcançar o objetivo da sprint, foram usadas ferramentas do design kit do HCD (IDEO, 2019). O Quadro 1 apresenta as sprints executadas por etapa do processo HCD e as respectivas ferramentas utilizadas. 
Quadro 1 - Sprints por etapa do processo HCD

\begin{tabular}{|c|c|c|}
\hline Etapa do HCD & Sprint & Ferramentas \\
\hline \multirow[t]{2}{*}{ Inspiração } & \#1 Descobrindo o desafio & $\begin{array}{l}\text { - Pesquisa Desk } \\
\text { - Brainstorming } \\
\text { - Desafio de Design }\end{array}$ \\
\hline & \#2 Aprofundando o entendimento & $\begin{array}{l}\text { - Imersão no Contexto } \\
\text { - Nuvem de Palavras }\end{array}$ \\
\hline \multirow{3}{*}{ Ideação } & \#3 Explorando Insights & $\begin{array}{l}\text { - Entrevista com Especialista } \\
\text { - Como nós Podemos? }\end{array}$ \\
\hline & \#4 Gerando e refinando ideias & $\begin{array}{l}\text { - Sessão de Cocriação } \\
\text { - Cardápio de Ideias }\end{array}$ \\
\hline & \#5 Materializando e avaliando ideias & $\begin{array}{l}\text { - Storyboard } \\
\text { - Protótipo }\end{array}$ \\
\hline \multirow{2}{*}{ Implementação } & \#6 Obtendo feedback & $\begin{array}{l}\text { - Análise de coorte } \\
\text { - Obter feedback }\end{array}$ \\
\hline & \#7 Evoluindo a solução & $\begin{array}{l}\text { - Protótipo (atualizado) } \\
\text { - Pitch }\end{array}$ \\
\hline
\end{tabular}

Fonte: Elaborado pelos autores (2020).

\subsection{Inspiração}

A etapa de Inspiração foi estruturada através de duas sprints: \#1 Descobrindo o desafio, com o objetivo de coletar informações sobre a temática do projeto e encontrar um desafio relevante para orientar o desenvolvimento de uma solução; e \#2 Aprofundando o entendimento, para imersão no contexto visando compreender o problema com clareza antes de propor uma solução.

A temática do projeto foi previamente definida e consistia no desenvolvimento de uma solução de inovação social digital relacionada ao ODS 13 da Agenda 2030 da ONU que envolve ações para combater os impactos da mudança do clima. A sprint \#1 iniciou com a pesquisa Desk que consistiu em usar o computador (desktop) para realizar buscas na $\mathrm{Web}$ para obter informações sobre a temática do projeto. O resultado foi a identificação das seguintes iniciativas sustentáveis:

- Bicycle Power Generation (ECYCLE, 2019): projeto desenvolvido na Universidade Politécnica da Califórnia (EUA), para geração de formas alternativas de energia sustentável e segura que possam atender uma grande quantidade de pessoas ao redor do mundo;

- Ohio Energy (OHIO ENERGY PROJECT, 2019): bicicletas conectadas a uma pequena placa elétrica possibilitam acender lâmpadas e fazem funcionar pequenos dispositivos eletrônicos, quando estas são usadas. A ideia é que os alunos observem as mudanças na intensidade da luz, conforme pedalam, e também as possíveis formas de gerar energia.

- EcoBike (ECOGREENS SOLUÇÕES SUSTENTÁVEIS, 2019): bicicleta que gera energia elétrica limpa. A bicicleta fica fixa enquanto as pessoas pedalam e usam o conceito de energia regenerativa, transformando a energia gerada pelas pedaladas em eletricidade. Além disso, disponibiliza uma plataforma interativa que entretém os usuários e ajuda na conscientização ambiental.

Em seguida, a equipe realizou uma sessão de brainstorming para definição do desafio de design. O resultado foi: "como juntar os aspectos de bem-estar e saúde, transformação de energia, ganhos econômicos, conscientização ambiental e diversão numa única solução?”.

A sprint \#2 iniciou com a imersão no contexto que consiste em ir ao encontro de um indivíduo ou comunidade e então observar como ele(s) vive(m), trabalha(m) e socializa $(m)$ de modo a melhor entender as pessoas no contexto do desafio de design. 
Desta forma, a equipe foi em espaços públicos onde funciona um projeto denominado Academia da Cidade (RECIFE, 2019) para observar e conversar com pessoas que realizam atividades físicas. Os principais termos citados pelos entrevistados podem ser observados através de uma Nuvem de Palavras (word cloud) (Figura 1).

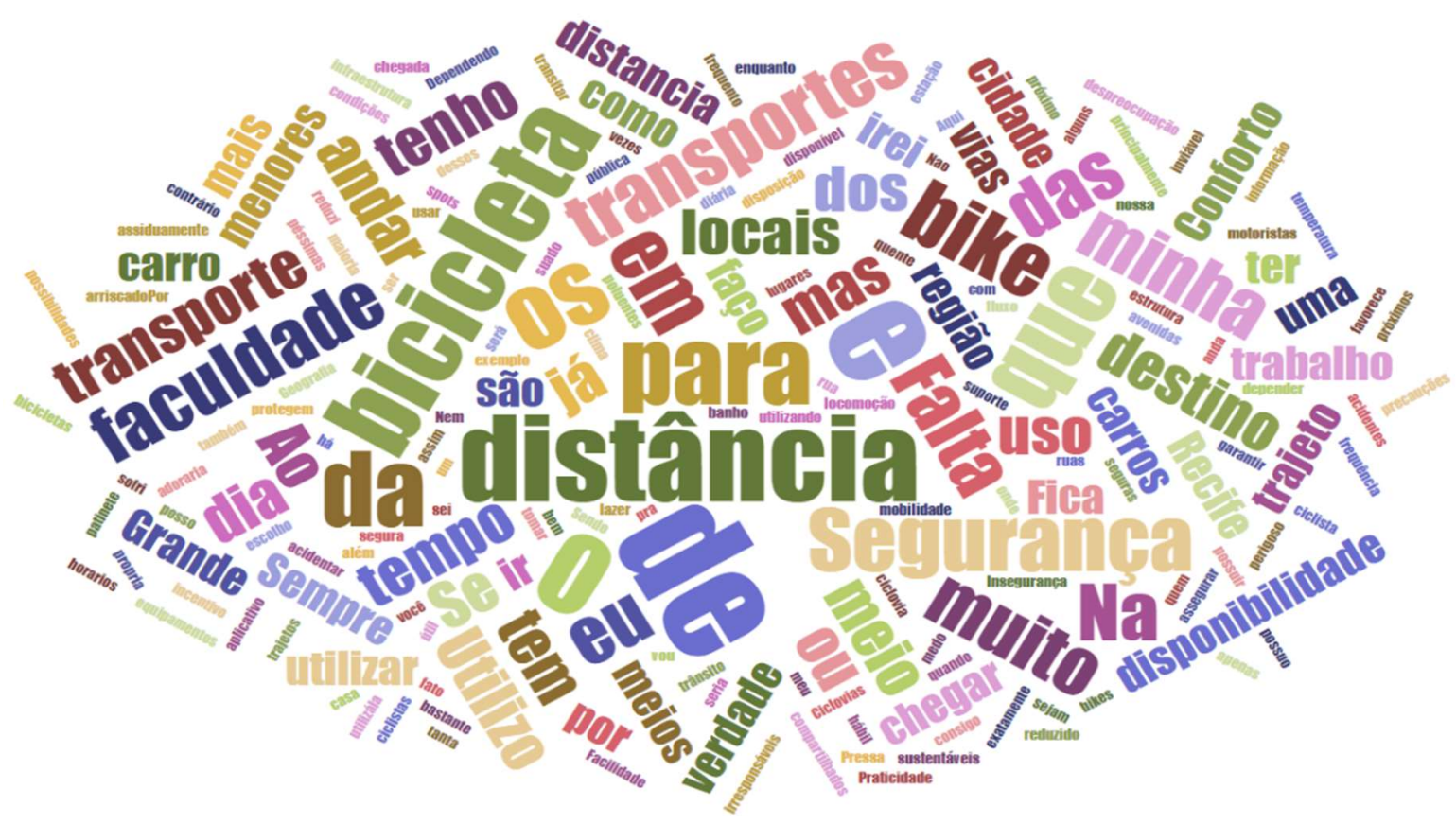

Figura 1 - Nuvem de Palavras com os principais termos citados.

Fonte: Elaborado pelos autores (2020).

\subsection{Ideação}

A etapa de Ideação foi estruturada através de três sprints: \#3 Explorando insights, com o objetivo de traduzir as descobertas da inspiração em oportunidades de inovação; \#4 Gerando e refinando ideias, para geração de ideias de solução de propriedade coletiva para atender os insights identificados; e \#5 Materializando e avaliando ideias, para prototipação das ideias visando a obtenção de feedback pelo público-alvo da ideia considerada mais promissora.

A sprint \#3 iniciou com a entrevista com especialista, que consistiu em formular perguntas e obter respostas de um especialista para que fosse possível adquirir uma visão técnica sobre o assunto e para identificação de possíveis oportunidades (insights) a serem exploradas.

A entrevista foi realizada com um engenheiro elétrico e foi possível identificar alguns importantes insights: a energia elétrica transformada a partir do movimento poderia ser usada para carregar pequenos aparelhos eletrônicos ou para introdução no sistema central de energia, gerando crédito "verde" a ser usado em descontos na conta de energia; uso do modelo de gamificação e recompensa para incentivar as pessoas a usarem uma solução de energia limpa e sustentável; e ausência de um modelo de negócios escalável para proporcionar a sustentabilidade do projeto.

A partir das informações obtidas até então, a equipe realizou uma sessão de brainstorming para usar a criatividade e identificar insights a partir de questões desafiadoras formuladas através do padrão: "como nós podemos ...?”. O resultado foi: “como nós podemos incentivar o exercício aeróbico das pessoas?”; "como nós podemos beneficiar o interesse coletivo a partir de ações individuais?"; "como nós podemos gerar energia limpa de modo a gerar crédito verde?”; " “como nós podemos recompensar as pessoas com comportamento sustentável?”.

A sprint \$4 iniciou com a sessão de cocriação que consiste em envolver ativamente as pessoas no processo coletivo e colaborativo de geração e avaliação de ideias. Desta forma, a equipe envolveu alguns estudantes universitários, usuários de serviços de bicicletas e patinetes compartilhados, para geração de ideias para atender os insights produzidos. O resultado foi a elaboração de um cardápio de ideias (Quadro 2). 
Quadro 2 - Cardápio de ideias

\begin{tabular}{|c|l|}
\hline $\mathbf{N}^{\mathbf{0}}$ & \multicolumn{1}{|c|}{ Descrição da Ideia } \\
\hline 1 & Bicicletas que armazenam a energia em estações (semelhante a Bike $\mathrm{PE}^{1}$ ) \\
\hline 2 & Bicicletas fixas que geram energia através da movimentação \\
\hline 3 & QR code nas bicicletas para vincular a algum aplicativo de bônus (milhas, etc.) \\
\hline 4 & Energia gerada pela pessoa, alimentaria a iluminação pública \\
\hline 5 & Fazer as bicicletas com materiais recicláveis \\
\hline 6 & Usar a bateria de carros antigos \\
\hline 7 & $\begin{array}{l}\text { Bicicletas feitas a partir de embalagens plásticas. Diminuição do uso do petróleo e redução da poluição } \\
\text { ambiental, bem como desastres ambientais marinhos }\end{array}$ \\
\hline 8 & $\begin{array}{l}\text { Bicicletas livres onde a pessoa cadastra o cartão metropolitano de transporte ou cartão que retira as } \\
\text { bicicletas, em uma aplicação que daria descontos na conta de luz ou bônus com a quantidade de } \\
\text { quilômetros rodados pela pessoa }\end{array}$ \\
\hline
\end{tabular}

Fonte: Elaborado pelos autores (2020).

A sprint \#5 iniciou com a elaboração de um storyboard que consiste de um protótipo de baixo nível, composto por quadros estáticos, usado para discutir e refinar uma ideia que pode ajudar a revelar quem irá usar, quando e como. A Figura 2 mostra um fragmento do storyboard produzido, o qual pode ser visualizado por completo em (https://bit.ly/2p8uabb).
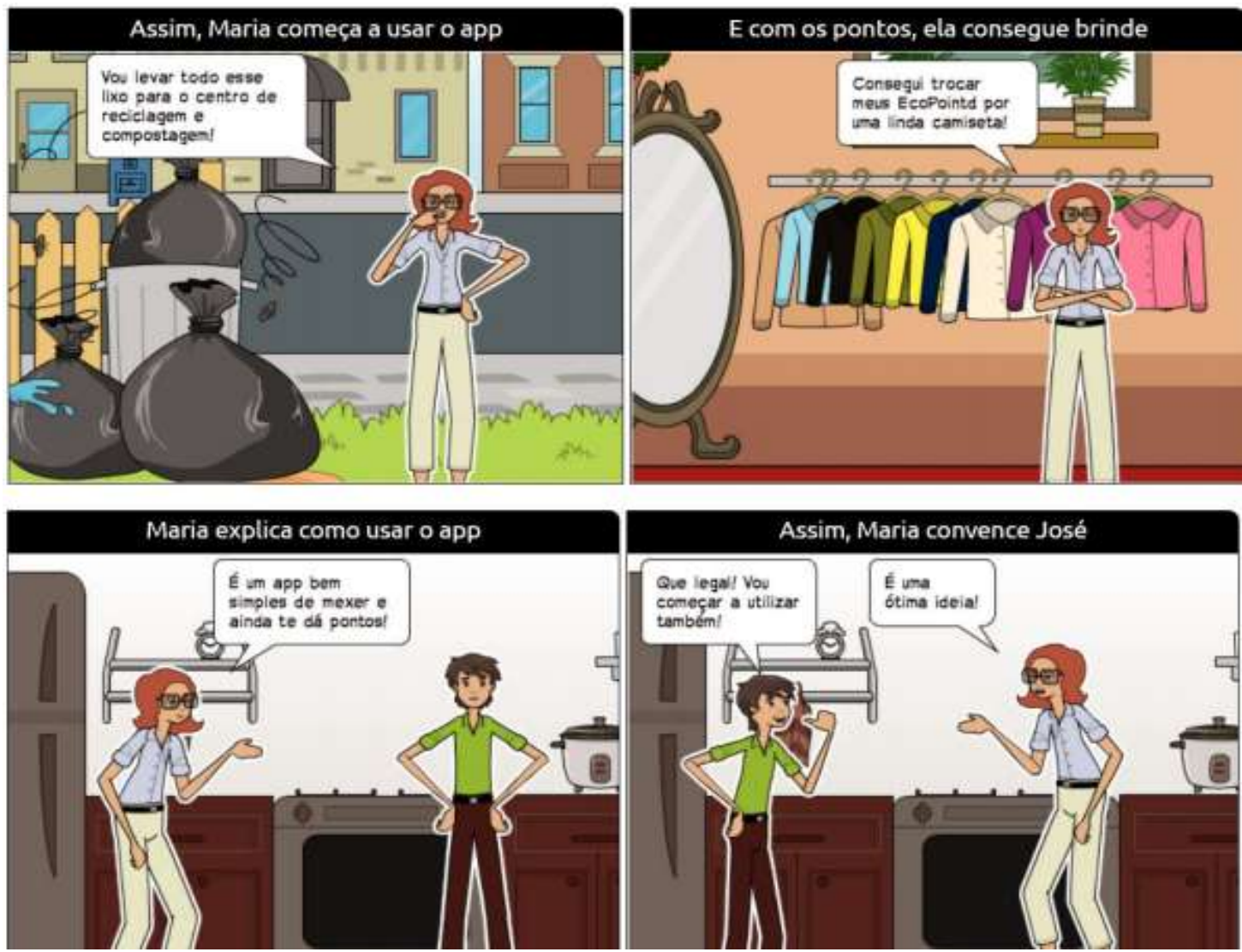

Figura 2 - Fragmento do Storyboard.

Fonte: Elaborado pelos autores (2020).

\footnotetext{
${ }^{1}$ https://bikeitau.com.br/bikepe/
} 
Em seguida foi desenvolvido um protótipo navegável da ideia de uma solução de inovação social digital para estimular o comportamento ambientalmente sustentável das pessoas por meio de um sistema de recompensa. A Figura 3 apresenta uma interface com o usuário (UI) do protótipo desenvolvido.

A UI retrata a funcionalidade do aplicativo de software, através da qual podem ser encontradas ações de comportamento sustentável pelas pessoas que podem ser transformadas em pontos para recompensas: compostagem, para transformação dos resíduos orgânicos em adubo natural; reciclagem, para conversão dos resíduos sólidos em outros elementos com potencial de uso, baseado no conceito de economia circular; e bikeenergy, para transformação de energia cinética produzida pelas pedaladas em energia elétrica.

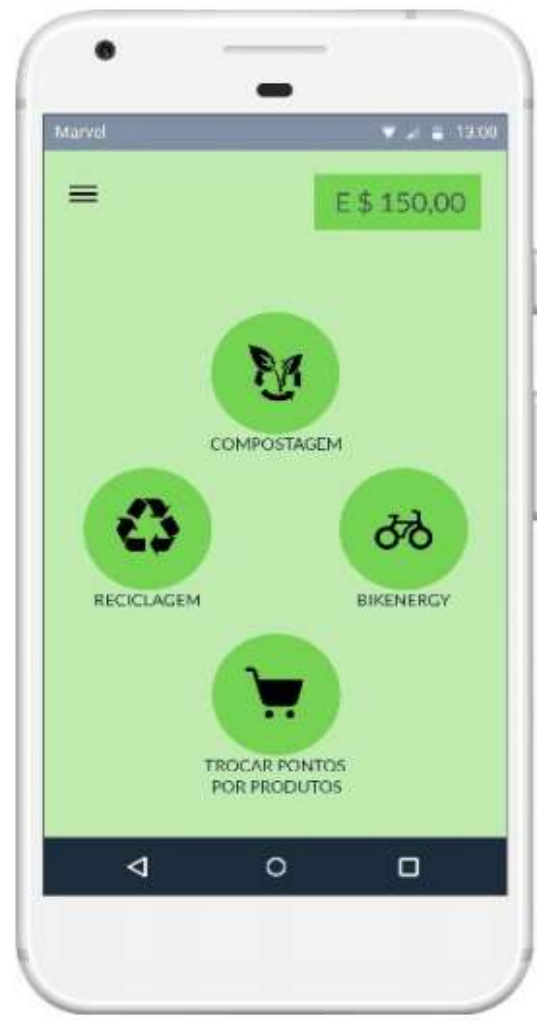

Figura 3 - UI do Protótipo.

Fonte: Elaborado pelos autores (2020).

\subsection{Implementação}

A etapa de implementação foi estruturada através de duas sprints: \#6 Obtendo feedback, com o objetivo de disponibilizar o protótipo desenvolvido para avaliação pelo público-alvo; e \#7 Evoluindo a solução, para evoluir o protótipo desenvolvido a partir do feedback obtido e apresentar o resultado final do projeto.

A sprint \#6 iniciou com a análise de coorte que consiste em disponibilizar o protótipo para grupos de usuários (coorte) de maneira independente e então avaliar os resultados. Para a análise de coorte foram identificados dois grupos de usuários com perspectivas diferentes com relação ao deslocamento para o trabalho e disponibilidade de tempo para outras atividades: (1) estudantes universitários que desempenham alguma atividade remunerada; (2) trabalhadores em vínculo acadêmico. Foi possível identificar que o primeiro grupo possui menor disponibilidade de tempo, já o segundo grupo tem maior flexibilidade nos horários. Ambos os grupos relataram interesse em adequar-se a uma vida com mais hábitos ambientalmente sustentáveis e que a solução proposta pode ser um catalisador para tal mudança.

Em seguida foi executada a técnica de obter feedback que consistiu na aplicação de um questionário eletrônico, respondido pelo público-alvo, sobre o uso do protótipo. A Figura 4 apresenta o gráfico com as respostas sobre o uso de um sistema de recompensas para incentivar o comportamento ambientalmente sustentável das pessoas, 
como o uso de transportes que não emitam poluentes, em uma escala de 1 (descordo totalmente) a 5 (concordo totalmente).

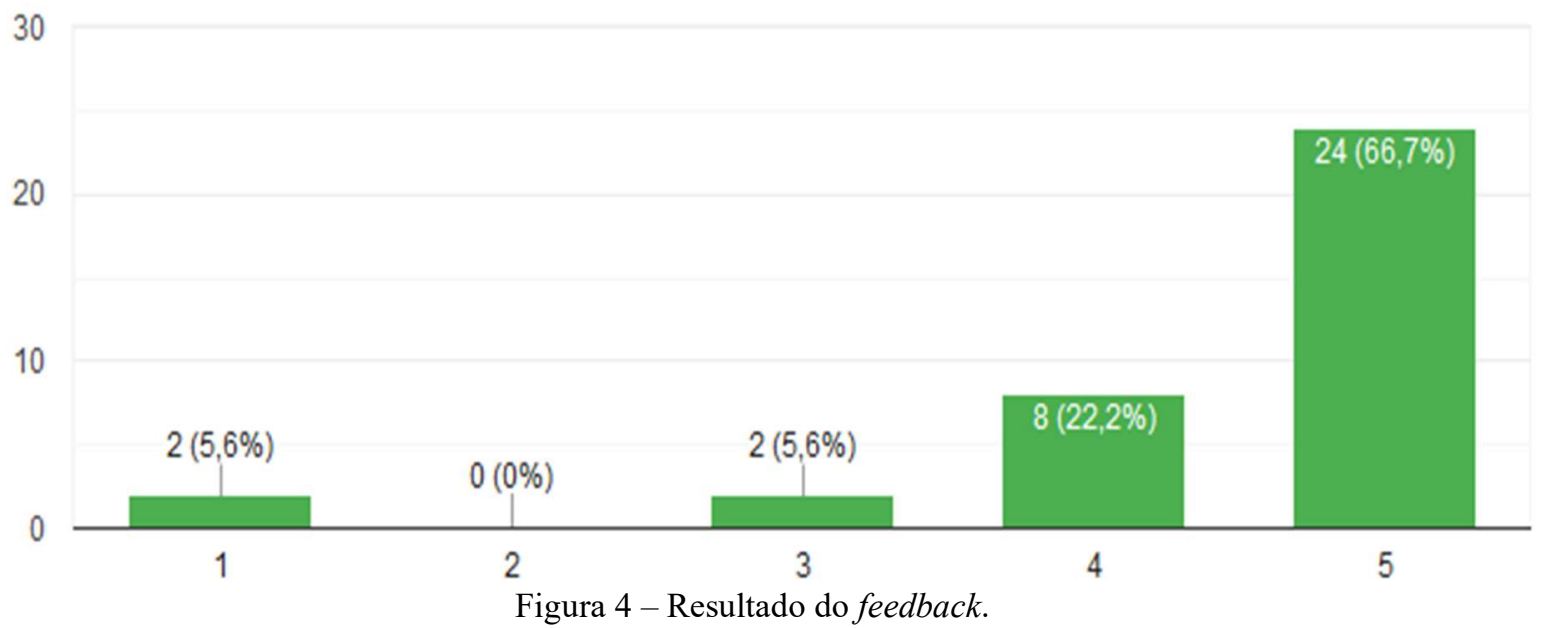

Fonte: Elaborado pelos autores (2020).

A sprint \#7 iniciou com a evolução do protótipo a partir do feedback obtido. A evolução do protótipo consistiu de uma versão enxuta funcional do aplicativo de software que demonstra a entrega de valor pela solução desenvolvida. A Figura 5 apresenta as principais UI do protótipo. A partir das UI é possível visualizar os caminhos que o usuário poderá percorrer dentro do aplicativo. O usuário necessitaria se deslocar até uma estação Eco+ para levar os resíduos para a compostagem ou reciclagem, ou para liberação de uma bicicleta adaptada que poderá fazer a conversão da energia cinética em energia elétrica. O aplicativo faria o controle do processo através de QRcode.

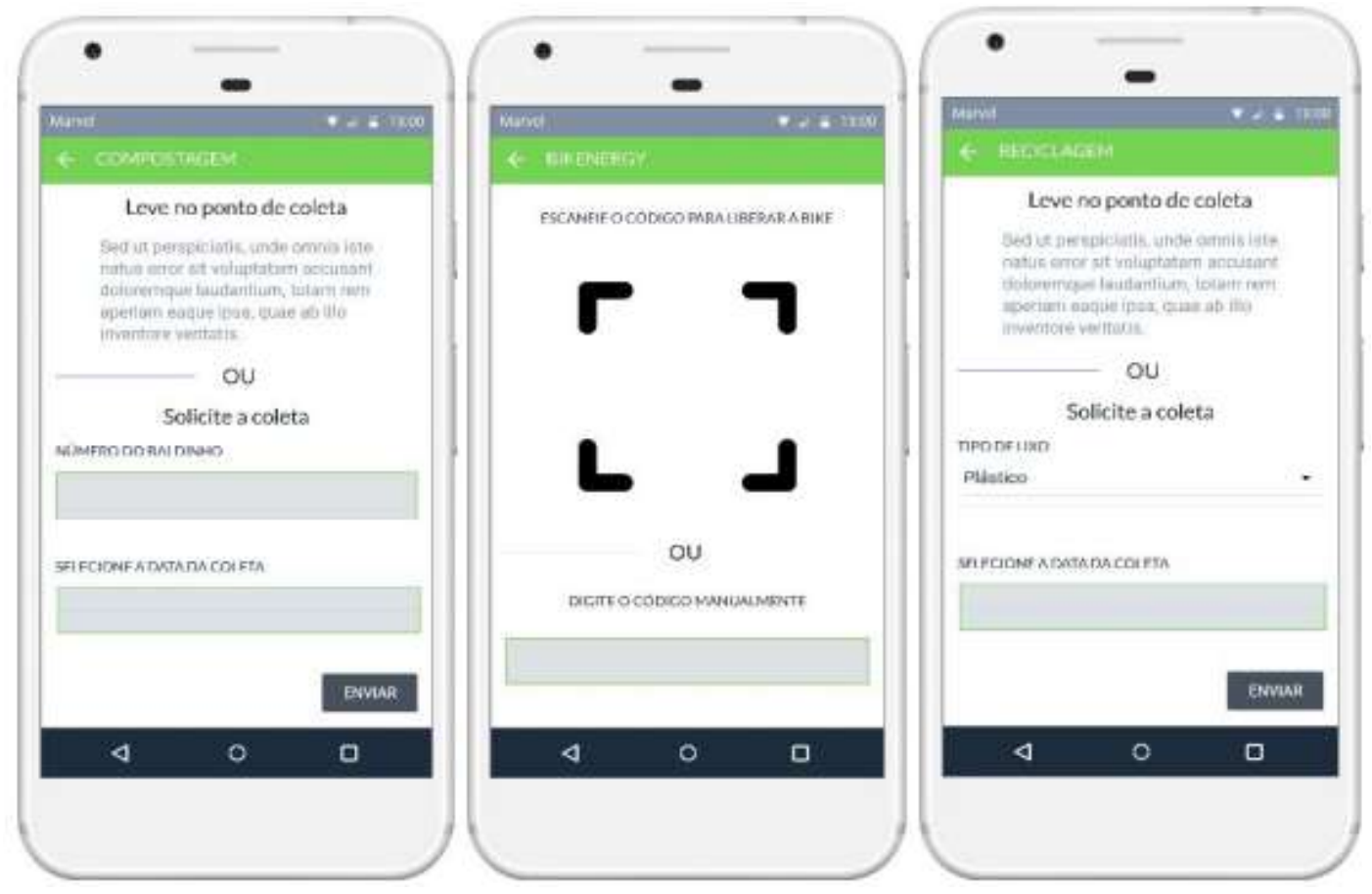

Figura 5 - UI da evolução do protótipo.

Fonte: Elaborado pelos autores (2020).

A sprint \#7, última do projeto, foi finalizada com a criação de um pitch sobre a solução desenvolvida. O pitch consiste em uma apresentação rápida e enxuta da solução proposta. A Figura 6 mostra os slides do pitch 
apresentado em um evento que reuniu diversos projetos desenvolvidos sobre a temática do ODS 13 da Agenda 2030 da ONU, na universidade que sediou o estudo.

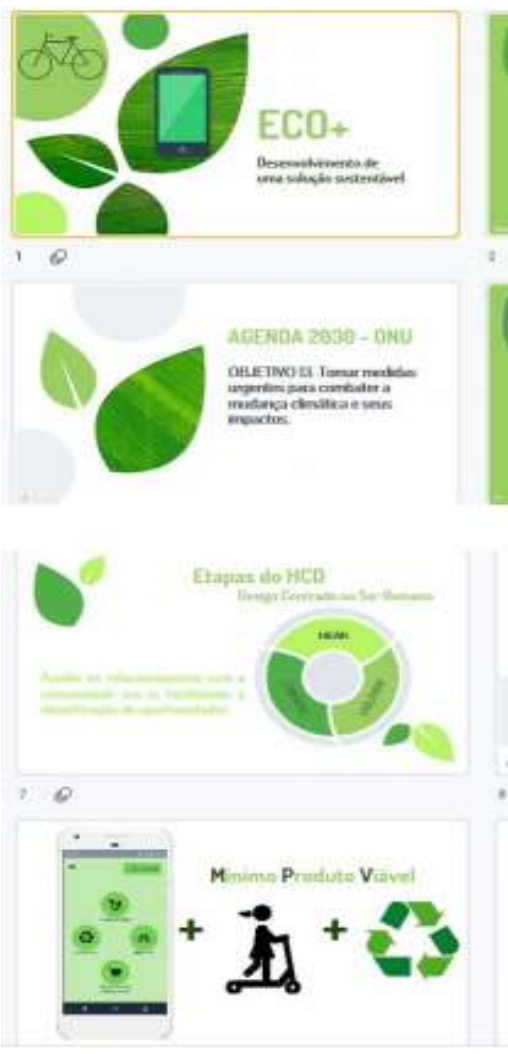

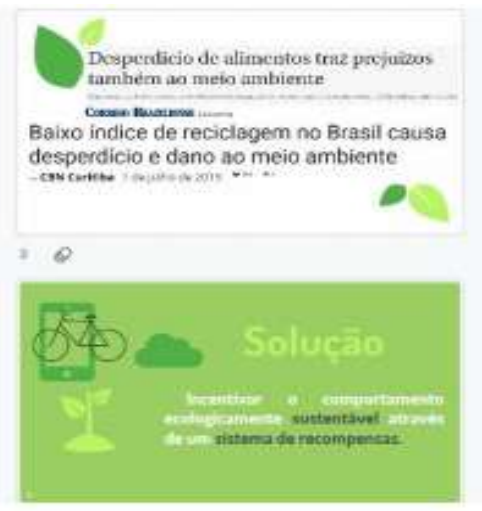
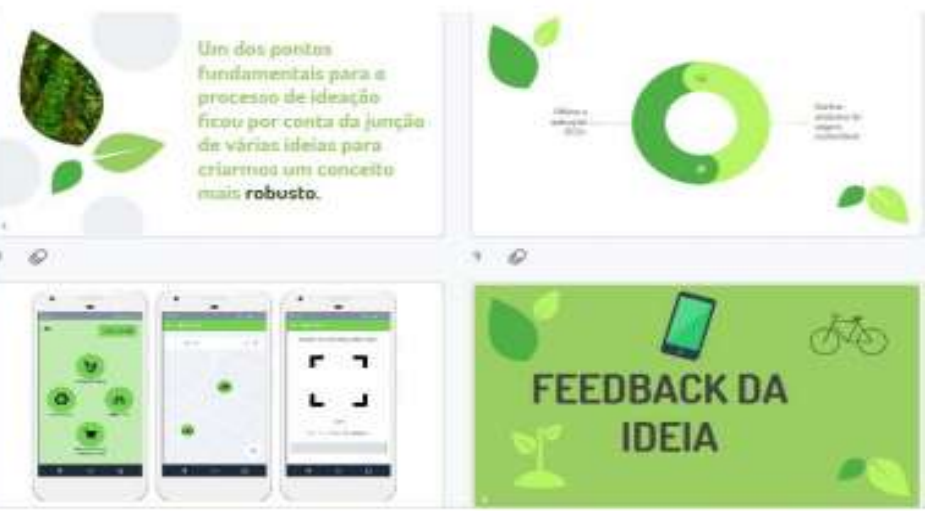

Figura 6 - Slides do Pitch.

Fonte: Elaborado pelos autores (2020).

\section{Conclusões}

Este artigo relata a experiência de aplicação do Design Centrado no Humano (HCD) para desenvolvimento de uma solução de inovação social digital relacionada ao Objetivo de Desenvolvimento Sustentável - ODS 13 da Agenda 2030 da ONU o qual se preocupa com medidas para combater os impactos da mudança do clima.

O HCD busca usar a criatividade para desenvolvimento de soluções para problemas reais da perspectiva das pessoas. O HCD é um processo enxuto composto pelas etapas de Inspiração, Ideação e Implementação. A Inspiração visa analisar o comportamento das pessoas e imergir no contexto para se ter um entendimento aprofundado antes da proposição de qualquer solução. A Ideação procura identificar oportunidades a partir das informações obtidas anteriormente e então gerar e avaliar ideias de solução. A Implementação serve para materializar e evoluir a ideia de solução que se mostrou mais promissora.

As etapas do processo HCD foram executadas através de iterações (sprints) com propósito bem definido e com prazo (deadline) não prorrogável. Para alcance do propósito de cada sprint, foram usadas ferramentas oriundas de abordagens de inovação. Ao todo foram executadas sete sprints, com duração de dez dias cada, e usadas quinze ferramentas diferentes. A aplicação do processo HCD, com etapas executadas por meio de sprints e com o auxílio de ferramentas de inovação de propósito específico, para desenvolvimento uma solução de inovação social digital, consistiu na contribuição científica deste trabalho.

A necessidade social que motivou o projeto foi as consequências das mudanças climáticas causadas pela atividade humana com grande produção de resíduos não aproveitados e com emissão de gases poluentes, principalmente por veículos automotivos. O desafio encontrado na inspiração e que norteou as demais etapas foi: “como estimular o comportamento ambientalmente sustentável pelas pessoas?”.

O resultado do projeto foi o design da plataforma Eco+ que fornece suporte tecnológico aos seguintes processos: o usuário pode realizar a coleta seletiva de resíduos e então levar até estações responsáveis por destiná-los à 
compostagem e/ou reciclagem; e o usuário pode usar bicicletas adaptadas para transformação da energia cinética e armazenagem da energia elétrica resultante.

O aplicativo de software prototipado possui como funcionalidades controlar o processo, através de mecanismos como QRCode, e fornecer pontos para os usuários que podem ser convertidos em recompensas, por exemplo, a troca por materiais ambientalmente sustentáveis. O design de um aplicativo de software para habilitar a inovação social de estímulo através de recompensas ao comportamento mais ambientalmente sustentável das pessoas consiste na contribuição técnica deste trabalho.

As limitações deste trabalho são o desenvolvimento apenas de um protótipo do aplicativo de software e na ausência de um modelo de negócios para garantir a sustentabilidade econômica da solução. As oportunidades de trabalhos futuros estão relacionadas às limitações apontadas: desenvolvimento de um produto final para o aplicativo de software e captação de parceiros, tais como, terceiro setor, organizações públicas, concessionárias de energia e empresas privadas para garantir o funcionamento da solução.

\section{Referências}

Anastacio, M. R., Cruz Filho, P. R., \& Marins, J. (2018). Social e Inovação Social no Contexto Brasileiro. Curitiba: PUCPRESS Editora Universitária Champagnat.

Baregheh, A., Rowley, J., \& Sambrook, S. (2009). Towards a multidisciplinary definition of innovation. Management decision. Vol. 47 No. 8, pp. 1323-1339. https://doi.org/10.1108/00251740910984578.

Bria, F., Gascó, M., Baeck, P., Halpin, H., Almirall, E., \& Kresin, F. (2015). Growing a Digital Social Innovation System for Europe. DSI Final Report.

Brown, T. (2009). Change by Design: How Design Thinking Transforms Organizations and Inspires Innovation. New York: HarperBusiness.

Ecogreens Soluções Sustentáveis. (2019). EcoBike. Recuperado a partir de http://www.ecogreens.com.br /home/index_site/ecobike.

Ecycle. (2019). Conceitos de bikes e acessórios para aproveitar a energia cinética das pedaladas. Recuperado a partir de https://www.ecycle.com.br.

IDEO. (2015). The Field Guide to Human-Centered Design. Design Kit.

IDEO. (2109). Design Kit. Recuperado a partir de http://www.designkit.org/methods.

IPCC. Intergovernmental Panel on Climate Change. (2007). Climate Change 2007: Synthesis Report. Contribution of Working Groups I, II and III to the Fourth Assessment Report of the Intergovernmental Panel on Climate Change. IPCC, Geneva, Switzerland, 104 pp.

Liedtka, J., Salzman, R., \& Azer, D. (2017). Design thinking for the greater good: Innovation in the social sector. Columbia University Press.

Mochizuki, Y., \& Bryan, A. (2015). Climate change education in the context of education for sustainable development: Rationale and principles. Journal of Education for Sustainable Development, 9(1), 4-26. https://doi.org/10.1177/0973408215569109.

Moulaert, F., Martinelli, F., González, S., \& Swyngedouw, E. (2007). Introduction: social innovation and governance in European cities: urban development between path dependency and radical innovation. European Urban and Regional Studies, 14(3), 195-209. https://doi.org/10.1177/0969776407077737.

Mulgan, G. (2012). Social innovation theories: Can theory catch up with practice?. In Challenge social innovation (pp. 19-42). Springer, Berlin, Heidelberg. https://doi.org/10.1007/978-3-642-32879-4_2.

Ohio Energy Project. (2019). Recuperado a partir de https://ohioenergy.org.

Oliveira, K. K., \& Souza, R. (2020). Mudanças climáticas na educação: um levantamento das práticas, ferramentas e tecnologias digitais. In Anais do XI Workshop de Computação Aplicada à Gestão do Meio Ambiente e Recursos Naturais (pp. 151-160). SBC. https://doi.org/10.5753/wcama.2020.11029.

ONU. Organização da Nações Unidas. (2015). Transformando Nosso Mundo: A Agenda 2030 para o Desenvolvimento Sustentável. Recuperado a partir de https://nacoesunidas.org/pos2015/agenda2030/.

Phills, J. A., Deiglmeier, K., \& Miller, D. T. (2008). Rediscovering social innovation. Stanford Social Innovation Review, 6(4), 34-43. 
Recife. (2019). Academia do Recife. Recuperado a partir de https://www.academiarecife.org.br.

Ribeiro, G. C., de Oliveira, R. F. F., Alencar, G. A., \& de Souza, R. A. C. (2018). Desenvolvimento de um aplicativo de alerta de alagamento utilizando os métodos do toolkit HCD. Boletim do Tempo Presente, (12). https://seer.ufs.br/index.php/tempopresente/article/view/11129.

Silva, J., Silva, T., Lima, M., \& Souza, R. (2019, July). Aplicação do Design Centrado em Humano para Desenvolvimento de uma Solução de Software baseada em Informações do Tempo e Clima. In Anais do X Workshop de Computação Aplicada a Gestão do Meio Ambiente e Recursos Naturais (pp. 107-116). SBC. https://doi.org/10.5753/wcama.2019.6425.

Visintainer, T., \& Linn, M. (2015). Sixth-grade students' progress in understanding the mechanisms of global climate change. Journal of Science Education and Technology, 24(2-3), 287-310. https://doi.org/10.1007/s10956-014-9538-0. 